production laitière à prendre en considération sera celle, soit de la vache à juger, soit de sa mère s'il s'agit d'un taureau ou d'une génisse, la note étant, dans ces deux derniers cas, réduite de moitié. De plus, l'attribution des prix les plus élevés sera réservée au classement de synthèse.

La fourniture de lait de brebis et de chèvre n'est pas à négliger.

On admet, pour les brebis, une production moyenne de 100 litres pendant les six mois que dure la lactation.

Parmi les principales races caprines, citons la race alpine, excellente laitière, dont il existe plusieurs variétés, et la race poitevine, composée d'individus robustes, moins bonne laitière, meilleure fromagère et excellente beurrière, fournissant en moyenne 400 à 700 litres de lait par an, soit 2 à 3 litres par jour.

(A suivre.)

\title{
STÉRILISATION PAR FLAMBAGE DES RÉCIPIENTS ET USTENSILES UTILISÉS EN LAITERIE
}

\author{
par
}

Georges RAY

Professeur honoraire (Tunis, Rennes, Grignọn)

Ex Chef de service à l'Institut intèrnational d'Agrieulture de Rome

L'application des méthodes classiques de stérilisation au traitement des récipients et ustensiles utilisés en laiterie soulève des problèmes dont la résolution est souvent difficile. On est en effet obligé de tenir compte de la nature des objets à stériliser, de leur grandeur, de leur forme, de la possibilité qu'on a de les manipuler plus ou moins aisément, si bien que la diversité des cas qu'il faut résoudre dans la pratique est très grande.

Comme les ressources dont on dispose habiuuellement sont modestes ou insuffisantes, particulièrement dans les exploitations agricoles, où l'eau et les moyens de chauffage manquent très souvent, on est conduit à mettre en œuvre des moyens simples et efficaces de réaliser à coup sûr une bonne stérilisation des ustensiles et du matériel laitier.

Cet objectif est atteint sans peine, si l'on applique la méthodede stérilisation dite par flambage, particulièrement en ce qui concerne le traitement du matériel des fermes, dont la stérilisation importe au premier chef, à qui désire produire ce que GorinI nomme des laits de pasteurisation facile.

\section{Conditions générales d'application du flambage}

On sait quels services rend le flambage, pour la stérilisation 
rapide et complète du petit matériel, dans les laboratoires de bactériologie, ainsi que dans les hôpitaux et eliniques. Rien ne s'oppose, en principe, à stériliser par flambage les ustensiles utilisés en laiterie, mais il faut toutefois fixer sans ambiguité les limites d'application de la méthode.

Il est évident tout d'abord que le flambage s'applique au traitement des surfaces aisément accessibles à la flamme, et en aucun cas à la stérilisation des objets carbonisables, tels que le bois (nu ou peint), le caoutchoue, les matières plastiques, la cellulose (papier, carton). Pourront être flambés tous les métaux nus, le verre, la porcelaine, les surfaces émaillées.

Comme conditions essentielles de réussite, nous retiendrons les point suivants :

10 Toute surface à flamber doit être préalablement nettoyée soigneusement et séchée. Tout dépôt aggloméré ou collé sur une paroi, ou dans un angle, ne pourrait être stérilisé par une flamme qu'en surface, et si l'on insistait, la carbonisation des matières organiques constituant le dépôt engendrerait de mauvaises odeurs, dues surtout à l'apparition de produits goudronneux;

$2^{\circ}$ Le brâleur utilisé pour le flambage doit produire une flamme bleue, incapable de laisser, après contact, un dépôt - même léger - de noir de fumée, et une mauvaise odeur. Il ne peut done s'agir que de brûleurs à admission d'air réglable, en fonction de l'arrivée du gaz combustible (gaz de ville, gaz butane, gaz d'alcool, et à la rigueur gaz de pétrole ou d'essence) ;

$3^{\circ} \mathrm{Au}$ cours du flambage, la flamme doit pouvoir être guidée à volonté, et réglée en intensité, de façon à permettre de lécher méthodiquement les surfaces à stériliser, sans laisser un seul point que la flamme n'ait pas touché. Ce travail demande donc beaucoup d'attention, et une conscience professionnelle absolue. Bâclé par un manœuvre inintelligent, ou négligent, le flambage serait sans effet, et ne donnerait qu'une fausse sécurité.

\section{Conditions pratiques d'application}

Nous allons préciser maintenant les conditions pratiques d'application du flambage, en examinant successivement le combustible, la production de la flamme, et enfin la technique du flambage.

Le combustible. Jusqu'à une période assez récente, les combustibles utilisables à la ferme étaient représentés par quelques produits liquides, comprenant :

Le pétrole et ses dérivés (essence et mazout).

L'alcool éthylique dénaturé type Régie.

La généralisation récente de la livraison de butane en bouteilles 
d'acier, a apporté dans ehaque ferme la possibilité d'utiliser un gaz combustible d'une grande commodité d'emploi. Il faut signaler également l'utilisation, dans certaines régions, assez étendues du Sud-Ouest, de gaz naturel, offrant les mêmes commodités d'emploi que le gaz de ville, et aussi l'utilisation possible dans les fermes convenablement équipées du gaz de fumier.

Le choix du combustible est guidé principalement par :

Les facilités d'approvisionnement et de transport ;

Le prix de revient;

Les servitudes eorrespondant aux précautions à prendre dans l'emploi, spécialement en ce qui concerne la limitation des accidents.

Production de la flamme. Répétons qu'il s'agit d'une flamme bleue, obtenue :

a) Dans le cas où l'on utilise les combustibles liquides, par un brûleur à gazéification, analogue aux nombreux appareils servant de lampes à souder, ou de lampes à braser, et de brûleurs très variés (réchauds de cuisine, brûleurs de laboratoires, d'ateliers, etc.);

b) Dans le cas où l'on utilise un combustible gazeux, par l'emploi de brûleurs de type Bunsen, Meker, Brandt, ou d'autres.

Toutefois, il y a lieu de tenir compte de certaines considérations dictées par la nécessité de faciliter l'application du flambage. Le brûleur doit répondre aux exigences suivantes :

1. Etre léger, facile à manipuler, à orienter dans tous les sens, et à ranger après usage.

2. Posséder un système d'arrêt instantané de la flamme et de rallumage automatique grâce à une veilleuse.

3. Posséder un réglage facile de l'arrivée d'air, par une virole à vis ou à glissière.

4. Etre muni de rallonges, coudes, et de dispositifs amovibles, permettant de changer, si besoin est, la forme de la flamme, notamment d'obtenir un papillon ou un dard.

On notera, à l'intention des producteurs intéressés, qu'il est facile de modifier un brûleur existant dans le commerce, en ajustant suivant les besoins, l'importance et la forme de la flamme, ainsi que la distance séparant la poignée de l'appareil de l'extrémité de la flamme. Les croquis donnés ci-joint (fig. 1 et 2 ) permettent de se rendre compte des réalisations qu'il est possible de tenter, en les confiant à un spécialiste, ou éventuellement à un bricoleur adroit.

\section{Technique du flambage}

Le passage de la flamme sur la surface à stériliser doit être rapide, et très soigneusement exécuté. Pour un ustensile déterminé, 
il sera indispensable d'adopter un ordre invariable, dans le flambage des surfaces. Prenons comme exemples, les cas les plus courants, savoir : le seau à traire, les bidons à lait, et enfin l'entonnoirfiltre. Voici comment nous conseillons de procéder.

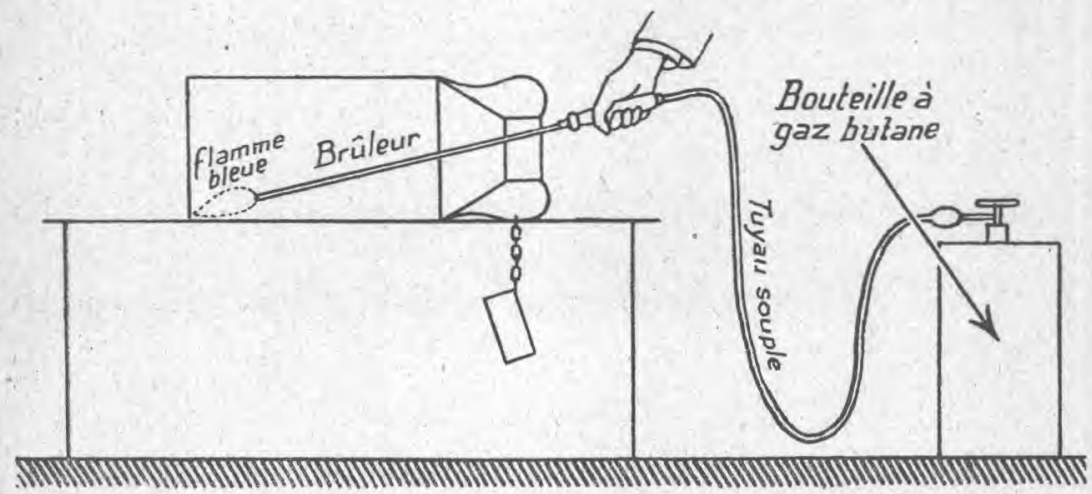

Fig. 1. - Représentation schématique du flambage d'un bidon.

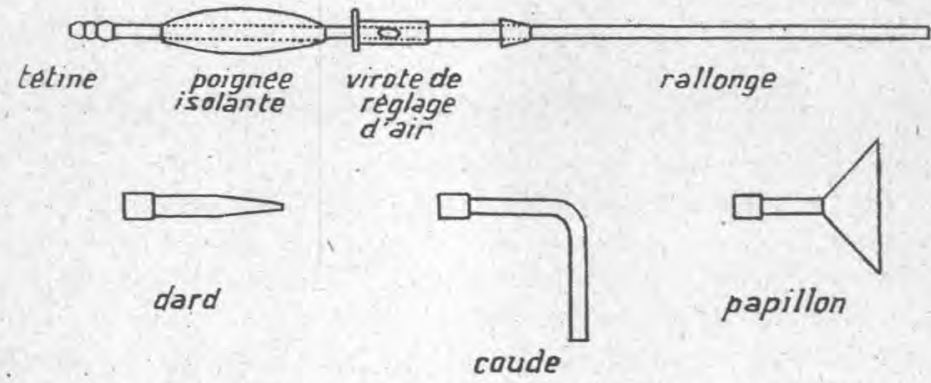

Fig. 2. - Schéma du brûleur portatif avec ses accessoires.

Flambage d'un seau à traire. Il est recommandable de procéder comme suit :

10 Flambage du fond, en partant du centre, et en promenant la flamme suivant une spirale;

$2^{\circ}$ Flambage de l'angle de raccord fond-paroi, exécuté avec une flamme en dard;

$3^{\circ}$ Flambage de la partie tronconique, en partant d'une génératrice (la ligne de soudure), et en allant alternativement de haut en bas, et de bas en haut;

$4^{0}$ Flambage du bord supérieur du seau, et de l'anse.

Flambage d'un bidon à lait. Le pot est couché sur une table. Opérer dans l'ordre suivant :

$1^{0}$ Flambage du fond (à flamme de forme normale) en partant du centre, et en promenant la flamme en spirale; 
20 Flambage de l'angle de raccord fond-paroi, à flamme en dard ;

$3^{\circ}$ Flambage de la partie cylindrique, en partant d'une génératrice (celle correspondant à l'une des anses); à cet effet; utiliser le coude et le bec papillon;

$4^{\circ}$ Flambage du tronc de cône (coude et papillon);

50 Flambage du col (coude et papillon);

6. Flambage du bouchon et de la chaîne (flamme normale);

$7^{\circ}$ Flambage des anses (ou des poignées).

Flambage de l'entonnoir-filtre. L'appareil étant démonté, et les pièces placées sur une table, procéder aux opérations suivantes :

10 Flambage du corps du filtre, en commençant par la partie conique, et en continuant par la partie cylindrique ;

$2^{\circ}$ Flambage des disques ou tamis, en les présentant à la flamme au moyen d'une pince de fer ;

30 Mise en place des disques ou tamis en se servant de la pince ;

4. Montage de l'entonnoir-filtre sur le col d'un bidon flambé, en ayant soin de couvrir l'entonnoir avec un disque de métal également flambé.

\section{Conseils généraux concernant l'organisation du travail}

L'opérateur travaille devant une table propre; il doit être vêtu. d'une blouse blanche récemment repassée. L'usage de gants est recommandable. Opérer sans brusquerie, et placer les ustensiles flambés ouverture en bas (dans le cas de vases) sur une surface nette, elle-même flambée, ouverture close dans le cas d'un bidon.

Il y a intérêt à réaliser le flambage immédiatement, ou peu de temps avant l'emploi des ustensiles.

\section{Contrôle de l'efficacité du flambage}

La perfection de la stérilisation assurée par le flambage dépend du soin apporté à l'opération, et du temps de contact de la flamme. Un temps très court suffit pour réaliser une stérilisation satisfaisante (quelques secondes). Toutefois, dans la pratique, avec un ouvrier d'habileté moyenne, on comptera :

Pour un seau à traire : 2 minutes environ.

Pour un bidon de 20 litres : 5 minutes environ.

Pour un entonnoir-filtre.: 3 minutes environ.

Il ne suffit d'ailleurs pas de consacrer un temps suffisant au flambage pour être certain d'obténir une bonne stérilisation. II est done indispensable d'entraîner le manipulateur chargé du flambage à l'exécution d'un travail extrêmement minutieux, dont rien ne doit le distraire. 
Le contrôle de la perfection du travail met en ouvre des numérations bactériennes, effectuées soit sur le produit du rinçage d'un ustensile ayant subi le flambage, soit sur un frottis réalisé par la méthode du faubert.

La première méthode me semble préférable, car elle donne un reflet beaucoup plus fidèle de l'état de toute la surface intérieure d'un bidon par exemple, et du degré de contamination qui pourra résulter du contact du lait avec les parois du récipient rempli.

Précisons que le bidon soumis à l'essai est rincé au moyen de $400 \mathrm{~cm}^{3}$ d'eau stérile tamponnée. Après vingt retournements dans le sens des aiguilles d'une montre, et vingt retournements en sens inverse, la solution de rinçage est recueillie dans un flacon stérile, et sert à la numération sur plaques.

On obtient invariablement des plaques nettes, dès que le temps consacré au flambage est suffisant, ce que j'ai obtenu en 4 à 5 minutes pour un bidon de 20 litres, en 2 à 3 minutes pour un seau à traire, en 4 à 5 minutes pour un entonnoir-filtre de type courant.

\title{
Application du flambage pour la stérilisation du matériel des laiteries
}

Tout matériel métallique dont les surfaces sont accessibles peut être stérilisé par flambage. C'est le cas par exemple pour les bacs, citernes, éléments de pompes, plaques de pasteurisateúrs, tuyauteries de diamètre suffisant, capsules métalliques pour bouteilles, etc.

Mais nous insistons sur le point suivant, déjà mis en vedette : c'est surtout dans les exploitations agricoles productrices de lait que la méthode de stérilisation par flambage, appliquée au traitement des seaux à traire, filtres, bidons à lait, éléments métalliques des machines à traire, apportera un progrès sensationnel, en réduisant fortement la teneur bactérienne des laits à l'arrivée à l'usine, et en permettant d'organiser efficacement la lutte contre les bactéries thermorésistantes.

\section{REVUE}

\section{L'INDUSTRIE LAITIËRE DANS LE MONDE}

\author{
par \\ G. GENIN \\ Ingénieur E.P.C.
}

\section{La production mondiale de beurre et de fromage}

L'U.S.D.A. indique que la production mondiale de beurre et de fromage a augmenté d'une façon sensible au cours de l'année 